\section{Impedanciometría esofágica para el diagnóstico de reflujo gastroesofágico en niños}

\section{RESUMEN}

El reflujo gastroesofágico es el paso retrógrado del contenido gástrico hacia el esófago, la enfermedad se produce cuando hay signos y síntomas. El método diagnóstico de elección ha sido la monitorización del pH intraesofágico; sin embargo, se han desarrollado nuevas técnicas para apoyar el diagnóstico. Una de ellas es la impedanciometría, que se basa en el registro de los cambios en la impedancia eléctrica en el lumen gastrointestinal provocada por el paso de un bolo (aire, alimento, saliva, contenido gastroduodenal). Con este estudio podemos valorar: el patrón del movimiento retrógrado y anterógrado, duración del bolo al deglutir o refluir, dirección y velocidad del movimiento esofágico y detectar cualquier episodio de reflujo, ya sea ácido o no ácido. El objetivo de esta revisión es evaluar el uso de este método en niños en cuanto a la técnica, indicaciones y beneficios para la vigilancia del $\mathrm{pH}$ intraesofágico.

Palabras clave: reflujo gastroesofágico, enfermedad por reflujo gastroesofágico, impedanciometría esofágica, reflujo gastroesofágico ácido, reflujo gastroesofágico no ácido.

\section{Esophageal impedance monitoring for the diagnosis of gastroesophageal reflux disease in children}

\section{ABSTRACT}

Gastroesophageal reflux is defined as the presence of gastric content towards the esophagus, and the gastroesophageal reflux disease is when it manifests with signs and symptoms. $\mathrm{pH}$ monitoring remains the primary diagnostic tool for detecting gastroesophageal reflux, but new technology has been developed to support this diagnose. The impedance-pH monitoring is based in the changes of the electric impedance at the gastrointestinal lumen given by the passing of the bolus (air, food, saliva, gastroduodenal content). With this study, we can evaluate the movement pattern antegrade or retrograde, bolus presence time, direction and velocity of bolus movement within the esophagus and detect any acid or non acid event. The objective of this revision is to evaluate this method in children, technique, indications and benefits of the impedance-pH monitoring.

Key words: Gastroesophageal reflux, gastroesophageal reflux disease, impedance monitoring, acid gastroesophageal reflux, nonacid gastroesophageal reflux.
Erick Manuel Toro-Monjaraz ${ }^{1}$ Esteban Gómez-Morales² Roberto Cervantes-Bustamante ${ }^{3}$ Jaime Ramírez-Mayans ${ }^{3}$

Flora Zárate-Mondragón ${ }^{3}$ Erika Montijo-Barrios ${ }^{3}$ José Francisco Cadena-León ${ }^{3}$ Monserrat Cazares-Méndez ${ }^{3}$ Martha López-Ugalde ${ }^{3}$

Servicio de Gastroenterología y Nutrición, Instituto Nacional de Pediatría (México).

${ }^{1}$ Coordinador general de la revisión.

${ }^{2}$ Recopilación de los datos, investigación de la literatura, crítica y redacción acerca del tema.

${ }^{3}$ Investigación de la literatura, crítica y redacción acerca del tema.
Recibido: 1 de noviembre del 2013

Aceptado: 9 de julio del 2014

Correspondencia: Dr. Erick Manuel Toro Monjaraz Departamento de Gastroenterología y Nutrición Pediátrica Instituto Nacional de Pediatría Insurgentes Sur 3700-C CP 04530 México, Distrito Federal Teléfono: 10840900 ext. 1288 emtoromonjaraz@hotmail.com

Este artículo debe citarse como Toro-Monjaraz EM, Gómez-Morales E, CervantesBustamante R, Ramírez-Mayans J, Zárate-Mondragón F, Montijo-Barrios E, et al. Impedanciometría esofágica para el diagnóstico de reflujo. Acta Pediatr Mex 2015;36:43-49. 


\section{INTRODUCCIÓN}

El reflujo gastroesofágico se define como el paso retrogrado del contenido gástrico hacia el esófago, es un proceso fisiológico que ocurre en niños sanos varias veces por día con una duración menor de 3 minutos. ${ }^{1}$ Hablamos de enfermedad por reflujo gastroesofágico cuando dicho reflujo provoca signos y síntomas. ${ }^{2}$

La vigilancia del $\mathrm{pH}$ intraesofágico ha sido aceptada, hasta el momento, como el procedimiento de elección para el diagnóstico del reflujo gastroesofágico en niños. ${ }^{3,4}$ Este estudio se lleva a cabo con un catéter que tiene un sensor de $\mathrm{pH}$ en su parte distal que se coloca $4 \mathrm{~cm}$ por arriba del esfínter esofágico inferior mediante la regla de Ströbel; el pH se vigila durante 24 horas y los programas encargados de procesar esta información normalmente muestran la frecuencia de reflujo ácido, tiempo total de $\mathrm{pH}$ por debajo de 4 y los episodios de reflujo que tienen una duración mayor a 5 minutos.

Existen diversos criterios para la evaluación de estos pacientes. La limitación de este estudio fue, precisamente, la determinación exclusiva de episodios de reflujo ácido.

\section{PRINCIPIOS DE LA IMPEDANCIOMETRÍA}

En 1991, Silny describió un nuevo método para evaluar la motilidad gastrointestinal basada en múltiples medidas de impedancia eléctrica. ${ }^{5}$ La impedanciometría se define como la proporción entre la corriente y el voltaje, medida en ohmios $(\Omega)$; es similar a la resistencia e inversamente proporcional a la conductividad eléctrica. ${ }^{6}$ El principio básico de esta técnica se asienta en el registro de los cambios en la impedancia eléctrica en el lumen gastrointestinal provocada por el paso de un bolo.

En la impedanciometría se aplican los siguientes principios: la conductividad del aire es casi de cero, por lo tanto, el aire tiene una conductividad eléctrica baja y una impedancia elevada. En contraste, la conductividad de un bolo (alimento, saliva, contenido gastroduodenal) es relativamente elevado. El aire, la pared esofágica y el cuerpo son componentes que funcionan como conductores alrededor del catéter y los electrodos.

El flujo de la electricidad entre los electrodos es posible debido a las cargas eléctricas producidas por la mucosa, submucosa y la capa muscular esofágica, permitiendo que la entrada de cualquier sustancia en el lumen esofágico (saliva, bolo de alimento o contenido gástrico regurgitado) produzcan cambios en la impedancia. ${ }^{7}$ La impedancia entre dos electrodos quiere decir que un canal de la impedancia cambia de características en la conductancia eléctrica dependiendo del material que rodea a estos electrodos y la fase de la onda de contracción. La impedancia disminuye durante al paso de un bolo con alta conductividad (alimento, saliva, contenido gástrico) y se incrementa durante el paso de aire o al momento de la contracción de la pared muscular del órgano.

Con registros de alta resolución en el esófago puede distinguirse entre el estado de reposo, de tránsito del bolo y de contracción muscular. Al utilizar canales múltiples en el catéter durante la impedanciometría se puede determinar la dirección del bolo y las contracciones musculares.

\section{MODO DE EMPLEO}

La impedanciometría puede realizarse de manera ambulatoria o bajo vigilancia hospitalaria. Sin embargo, se considera que la manera ambulatoria es la ideal ya que el paciente se desenvuelve en su ambiente cotidiano, permitiendo observar las características y cambios en el estudio de una manera más real, inclusive al momento de reali- 
zar actividad física que puede ser un detonante de los síntomas.

En cuanto a la preparación del paciente es necesario un ayuno de 3 horas antes de la colocación del catéter para evitar vómitos y aspiración. Durante el estudio la dieta debe ser la habitual; sin embargo, es necesario evitar alimentos o bebidas muy calientes o muy fríos, jugos ácidos y bebidas carbonatadas, ya que pueden interferir con la sensibilidad de la prueba. ${ }^{8}$

El estudio consta de dos elementos fundamentales: el catéter y el amplificador que va a reportar los datos obtenidos. Los catéteres a utilizar dependerán de la edad del paciente; los más utilizados son el infantil ( 0 a 2 años), el pediátrico ( 2 a 10 años) y el adulto ( $\geq 10$ años). Cada catéter tiene un diámetro de 2.13 mm $(6.4$ French) y cuenta con 7 sensores distribuidos a lo largo del mismo, con una forma de anillo cilíndrico de $4 \mathrm{~mm}$. El segmento que se encuentra entre cada par de anillos corresponde a un canal medible en la impedanciometría. Al constar de 7 sensores se obtienen 6 lecturas a diferentes niveles del esófago. El electrodo del $\mathrm{pH}$ se coloca en el centro del sensor de impedancia más distal, aunque también se cuenta con catéteres con 2 puntos para la medición del $\mathrm{pH} .{ }^{6,8,9}$

La colocación del catéter es fundamental para obtener una prueba adecuada. El catéter se debe colocar de manera intranasal hasta el esófago hasta que se el sensor de $\mathrm{pH}$ esté a $5 \mathrm{~cm}$ del esfínter esofágico inferior; así, los segmentos de medición de impedanciometría estarán 3, 5, 7 y $9 \mathrm{~cm}$ por arriba del esfínter esofágico inferior en la parte distal y a 15 y $17 \mathrm{~cm}$ en el esófago proximal. Hay otras variantes para la adecuada colocación del catéter como la fórmula de Strobe ${ }^{10}$ para pacientes menores de un año: distancia entre narinas y esfínter esofágico inferior $(\mathrm{cm})=(0.252 \times$ estatura en $\mathrm{cm})+5$.
En pacientes mayores la manera más exacta es mediante manometría o endoscopia. La colocación correcta del catéter debe ser corroborada con radioscopia durante todo un ciclo respiratorio ya que la punta del electrodo se moviliza durante la inspiración y la espiración. El uso de gel no altera los resultados del estudio, sólo se debe de evitar que toque el electrodo distal (canal del $\mathrm{pH}$ ) ya que puede disminuir su precisión. En la Figura 1 se muestra la posición correcta de la sonda de impedanciometría. ${ }^{11}$

En cuanto al uso de medicamentos durante el estudio se recomienda que se suspendan según sea el medicamento empleado: los inhibidores de la bomba de protones se deben suspender 7 días, antihistamínicos 3 días y procinéticos 2 días antes de la prueba, aunque en la edad pediátrica no se cuenta con estudios en los que haya reportes de cambios con o sin el uso de estos medicamentos. ${ }^{12}$

Se recomienda que durante el estudio no se utilice chupón ni se masque chicle ${ }^{13}$ porque aumentan la producción de saliva e incrementan

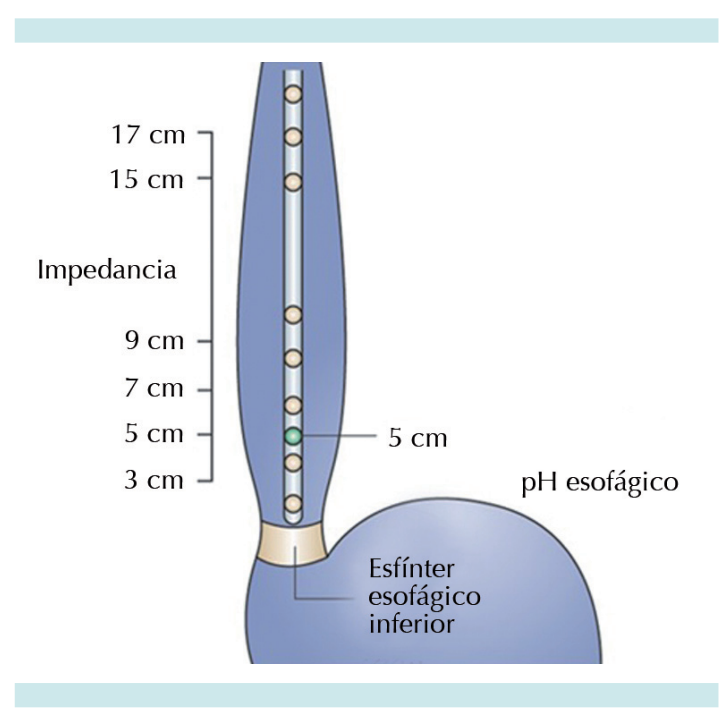

Figura 1. Colocación correcta de la sonda de impedanciometría. Modificada de Gastroesophageal reflux monitoring: $\mathrm{pH}$ and impedance. ${ }^{11}$ 
el número de degluciones en el paciente, lo que hace más compleja la detección de datos de reflujo retrógrado.

\section{UTILIDAD EN NIÑOS}

La impedanciometría ha permitido nuevos descubrimientos en la fisiología y fisiopatología de la función gastrointestinal en pacientes sanos y enfermos: patrón del movimiento retrógrado y anterógrado del bolo, duración del bolo al deglutir o refluir, dirección y velocidad del movimiento esofágico.

Dentro del área pediátrica parece que esta técnica ofrece mayores beneficios y ventajas en cuanto al entendimiento y valoración en pacientes con reflujo gastroesofágico. Al no ser dependiente de un estado ácido, como en la vigilancia del $\mathrm{pH}$ intraesofágico, la impedanciometría puede detectar cualquier episodio de reflujo ya sea ácido o no ácido. Así mismo, detecta la dirección del bolo y permite así evaluar la función motora del esófago. En el Cuadro 1 se hace una comparación entre la pHmetría convencional y la $\mathrm{pH}$ impedanciometría. ${ }^{14}$

La impedanciometría puede utilizarse junto con otros estudios de manera simultánea, como es el caso de impedanciometría más vigilancia del pH intraesofágico o junto con manometría; o de manera complementaria como la polisomnografía

Cuadro 1. Comparación entre la pH-metría convencional y la $\mathrm{pH}$-impedanciometría

\begin{tabular}{lll}
\hline Parámetro & $\begin{array}{l}\text { Ph-metría } \\
\text { convencional }\end{array}$ & $\begin{array}{l}\text { Impedanciome- } \\
\text { tría }\end{array}$ \\
RGE ácido & Sí & Sí \\
RGE no ácido & No & Sí \\
Reflujo de gas & No & Sí \\
Altura del reflujo & 1 a 2 niveles & 6 a 7 niveles \\
Aclaramiento del bolo & No & Sí \\
RGE posprandial & No & Sí
\end{tabular}

RGE: reflujo gastroesofágico. para detectar alteraciones respiratorias durante el sueño (apneas, desaturaciones). ${ }^{15,16}$

\section{INTERPRETACIÓN DE RESULTADOS}

En la impedanciometría se habla de reflujo cuando la impedancia intraluminal cae 50\% con respecto de la línea basal y progresa de manera retrógrada a través de 2 o más canales distales. Se considera evento ácido cuando el pH esofágico disminuye y permanece en $\geq 4$ por al menos 5 segundos; por último, un episodio no ácido es cuando el $\mathrm{pH}$ se incrementa, se mantiene sin cambio o disminuye una unidad de $\mathrm{pH}$ mientras se mantenga por arriba de 4 .

\section{Valores normales}

Existe sólo un estudio en prematuros realizado por López Alonso, ${ }^{17}$ por lo que en niños más grandes se usan como parámetros de referencia los obtenidos por Shay y Zerbib..$^{9,18}$ En el servicio de Gastroenterología y Nutrición en el Instituto Nacional de Pediatría utilizamos los valores obtenidos por Shay. En el Cuadro 2 se describen estos valores.

Análisis de los síntomas

Dentro del análisis de datos en la impedanciometría es de vital importancia analizar los síntomas que se asocian con cada episodio de reflujo, ya sea ácido o no ácido. Para ello se han descrito 3 medidas:

1. Índice de síntomas: se define como el porcentaje de episodios de síntomas asociados con el reflujo. El valor normal es menor a 50\% para cada síntoma. ${ }^{14,19}$

2. Índice de sensibilidad de síntomas: se define como el porcentaje de episodios de reflujo asociados con los síntomas. Valores por arriba de $10 \%$ se consideran positivos. ${ }^{14}$ 
Toro-Monjaraz EM et al. Impedanciometría esofágica en niños

Cuadro 2. Valores obtenidos por Shay

\begin{tabular}{lcccccccc}
\hline & \multicolumn{3}{c}{ Eventos de reflujo distal } & \multicolumn{5}{c}{ Eventos de reflujo proximal } \\
& Total & Ácido & Débilmente ácido & No ácido & Total & Ácido & Débilmente ácido & No ácido \\
& 73 & 55 & 26 & 1 & 31 & 28 & 12 & 1 \\
Total & 67 & 55 & 24 & 1 & 29 & 25 & 11 & 1 \\
De pie & 7 & 5 & 4 & 0 & 3 & 2 & 1 & 0 \\
Acostado & 7 & & & & & &
\end{tabular}

3. Probabilidad de asociación de síntomas: determina la significación estadística de la correlación reflujo-síntoma y se calcula dividiendo los datos de 24 horas en intervalos consecutivos de 2 o 5 minutos. Un valor por arriba de $95 \%$ es positivo. ${ }^{14}$

En la Figura 2 se muestra un evento de reflujo ácido y en la Figura 3 un evento no ácido.

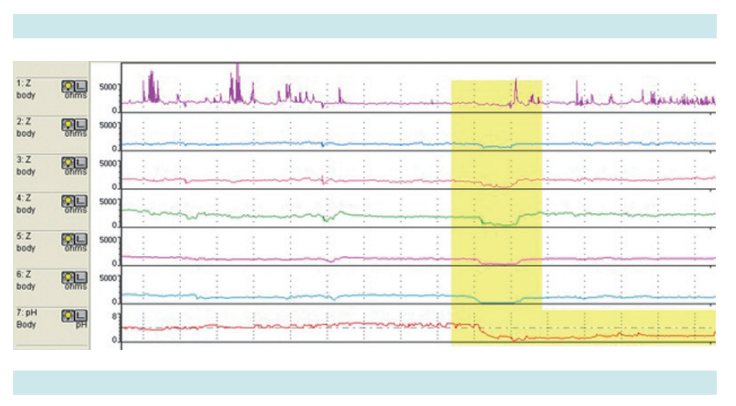

Figura 2. Gráfica de impedanciometría demostrando la presencia de reflujo (progresión anterógrada ascendente) catalogado como ácido por la caída del $\mathrm{pH}$ por debajo de 4 .

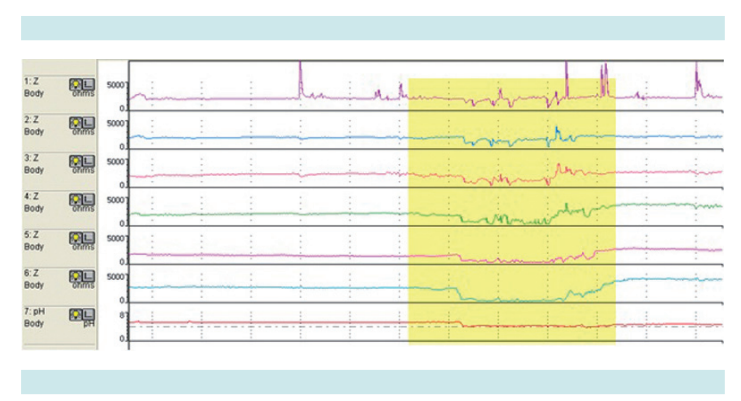

Figura 3. Gráfica de impedanciometría demostrando la presencia de reflujo no ácido ya que mantiene el $\mathrm{pH}$ dentro de rangos normales.

\section{COMPLICACIONES DEL ESTUDIO}

Las causas y el número de complicaciones son semejantes a las encontradas en la pHmetría convencional; es decir, son bajas. Incluyen las fallas técnicas del equipo, la mala colocación de los catéteres y daños a la mucosa como sangrado o laceración; esto siempre se debe de explicar de manera detallada a los familiares del paciente. ${ }^{14}$

\section{ESTUDIOS REALIZADOS EN NIÑOS}

Varios estudios han mostrado la ventaja que tiene la combinación de la impedanciometría y la vigilancia del $\mathrm{pH}$ ( $\mathrm{pH}$-imepdanciometría esofágica) en la práctica pediátrica cotidiana. En un estudio realizado en Alemania, en cuatro centros pediátricos, se efectuó $\mathrm{pH}$-impedanciometría en 700 niños, 270 resultaron positivos y de éstos $101(37 \%)$ tuvieron resultados anormales tanto en la impedanciometría como en la vigilancia del $\mathrm{pH}$ intraesofágico durante 24 horas: 18\% mostró resultados anormales sólo en pH-metría intraesofágica de 24 horas y $45 \%$ mostró resultados anormales en impedanciometría. ${ }^{20}$ Lo anterior demuestra la superioridad de la vigilancia mediante $\mathrm{pH}$-impedanciometría en la evaluación del reflujo.

En un estudio español realizado por Tolin y sus colaboradores ${ }^{21}$ se compararon los hallazgos mediante vigilancia del $\mathrm{pH}$ intraesofágico durante 24 horas y la impedanciometría en 49 niños: $18 \%$ mostraron sólo $\mathrm{pH}$-metría intraesofágica de 24 horas positiva mientras que $39.2 \%$ mostraron impedanciometría positiva. Además, en este 
estudio se evaluó la asociación con síntomas respiratorios y el índice de síntomas fue de $6.7 \%$.

Rosen y su grupo realizaron un estudio, en el hospital de niños de Boston, en el que se les mostraba a diferentes médicos el resultado de una vigilancia del $\mathrm{pH}$ intraesofágico y el tratamiento que administrarían. Posteriormente, les enseñaban el resultado de la impedanciometría y les preguntaban si realizarían algún cambio en el manejo inicial. Los resultados fueron: cambio de manejo en $25 \%$ de los casos sugiriendo un papel importante de la impedanciometría en la práctica clínica. $^{22}$

En Denver, Condino y sus colegas ${ }^{23}$ buscaron la proporción de reflujo gastroesofágico ácido y no ácido utilizando con herramienta de diagnóstico a la impedanciometría, así como la determinación del índice de correlación con los síntomas. Sus hallazgos fueron que en la impedanciometría se encontraron 1890 eventos de reflujo, comparados con 588 obtenidos mediante la vigilancia del $\mathrm{pH}$ intraesofágico: $47 \%$ ácidos y 53\% no ácidos. En cuanto a la relación con los síntomas $24.6 \%$ se correlacionó con eventos no ácidos y $25.2 \%$ con eventos ácidos. Concluyeron que la impedanciometría detecta más eventos de reflujo que la vigilancia del pH intraesofágico y que sus resultados correlacionan más con los síntomas.

\section{CONCLUSIONES}

La impedanciometría multicanal combinada con la monitorización del pH intraesofágico promete convertirse en el método de elección para el diagnóstico del reflujo gastroesofágico en niños. Actualmente la mayor limitante es la falta de parámetros normales en niños. Consideramos que cuando se cuente con la posibilidad de evaluación por medio de impedanciometría ésta se debe de realizar ya que ofrece mucho más datos sobre el tipo de reflujo y sobre las características del mismo.

\section{REFERENCIAS}

1. Sherman PM, Hassall E, Fagundes-Neto U, Gold BD, Kato S, Koletzko S, et al. A global evidence-based consensus on the definition of gastroesophageal reflux disease in children. Am J Gastroenterol 2009;104:1278-95.

2. Ramírez Mayans J, de la Torre Mondragón L, Azuara Fernández $\mathrm{H}$, Cervantes Bustamante R, Coran Arnold G, Berchi $\mathrm{F}$, et al. Consenso médico quirúrgico para el manejo de niños con reflujo gastroesofágico. Rev Gastroenterol Mex 2002;68(3):223-234.

3. Colletti RB, Chrisie DL, Orenstein. SR Indications for pediatrics esophageal $\mathrm{pH}$ monitoring. Statement of the North American Society for Pediatric Gastroenterology and Nutrition (NASPGN). J Pediatr Gastroenterol Nutr 1995;21:253-262.

4. VandenplasY, BelliD, Boige N. A standardized protocol for themethodology of esophageal $\mathrm{pH}$ monitoring and interpretation of the data for the diagnosis of gastroesophageal reflux. Statement of the European Society of Pediatric Gastroenterology and Nutrition (NASPGHAN) (1992). J Pediatr Gastroenterol Nutr 1992;14:467-471.

5. Silny J. Intraluminal multiple electric impedance procedure for measurement of gastrointestinal motility. Neurogastroenterol Motil 1991;3:151-162.

6. Zentilin P, Dulbecco P, Savarino E, Giannini E, and Savarino V. Combined multichannel intraluminal impedance and phmetry: a novel technique to improve detection of gastrooesophageal reflux. Literature review. Digestive and Liver Disease 2004;36(9):565-9.

7. D Sifrim, D Castell, J Dent, P J Kahrilas, Gastro-oesophageal reflux monitoring: review and consensus report on detection and definitions of acid, non-acid, and gas reflux. Gut 2004;53:1024-1031.

8. Hayat M. Mousa, Rachel Rosen, Frederick W. Woodley. Esophageal Impedance Monitoring for Gastroesophageal Reflux. JPGN 2011;52:129-139.

9. F. Zerbib, S. Bruley Des Varannes, S. Roman, P. Pouderoux, Normal values and day-to-day variability of 24-h ambulatory oesophageal impedance-pH monitoring in a BelgianFrench cohort of healthy subjects. Aliment Pharmacol Ther 2005;22:1011-102.

10. Strobel CT, Byrne WJ, Ament ME. Correlation of esophageal lengths in children with height: application to the Tuttle test without prior esophageal manometry. J Pediatr 1979;94:81-4.

11. Radu Tutuian, M.D. and Donald O. Castell, M.D. Gastroesophageal reflux monitoring: $\mathrm{pH}$ and impedance. $\mathrm{Gl}$ Motility online, May, 2006. doi:10.1038/gimo31.

12. Maine I, Tutuian R, Shay S. Acid and non-acid suppressive therapy in patients with persistent symptoms despite acid suppressive therapy: a multicenter study using combined ambulatory impedance-pH monitoring. Gut 2006;55:1398402. 
Toro-Monjaraz EM et al. Impedanciometría esofágica en niños

13. Smoak BR, Koufman JA. Effects of gum chewing on pharyngeal and esophageal $\mathrm{pH}$. Ann Otol Rhinol Laryngol 2001;110:1117-9.

14. Adria A. Condino, Judith Sondheimer, Zhaoxing Pan. Evaluation of Infantile Acid and Nonacid Gastroesophageal Reflux Using Combined pH Monitoring and Impedance Measurement. Journal of Pediatric Gastroenterology and Nutrition 2006;42:16-21.

15. Radu Tutuian, MD; Inder Mainie, MRCP; Amit Agrawal, MD. Nonacid Reflux in Patients With Chronic Cough on Acid-Suppressive Therapy. Chest 2006;130:386-391.

16. Rosen $R$, Nurko $S$. The importance of multichannel intraluminal impedance in the evaluation of children with persistent respiratory symptoms. Am J Gastroenterol 2004;99:2452-8.

17. Manuel López-Alonso, Maria Jose Moya, Jose Antonio Cabo, Juan Ribas, Maria del Carmen Macías, Jiry Silny, et al. Alkaline Twenty-Four-Hour Esophageal Impedance-pH Monitoring in Healthy Preterm Neonates: Rate and Characteristics of Acid, Weakly Acidic, and Weakly Gastroesophageal Reflux. Pediatrics 2006;118:e299.

18. Shay $S$, Tutuian $R$, Sifrim $D$, et al. Twenty-four hour ambulatory simultaneous impedance and $\mathrm{pH}$ monitoring: a multicenter report of normal values from 60 healthy volunteers. Am J Gastroenterol 2004;99:1037-43.
19. Wiener GJ, Richter JE, Copper JB, et al. The symptom index: a clinically important parameter of ambulatory 24-hr esophageal pH monitoring. Am J Gastroenterol 1988;83:358-61.

20. Denisa Pilic, MD, Thorsten Fröhlich, MD, Frank Nöh, MD. Detection of Gastroesophageal Reflux in Children Using Combined Multichannel Intraluminal Impedance and $\mathrm{pH}$ Measurement: Data from the German Pediatric Impedance Group. J Pediatr 2011;158:650-4.

21. Tolin-Henrnani M, Crespo-Medina M, Luengo-Herrero V., Mártinez López C, Salcedo Posadas A, Alvarez Calatayud G, Comparación entre $\mathrm{pH}$-metría convencional e impedanciometría intraluminal multicanal en niños con patología respiratoria, An Pediatr Barc 2012;77(2):103-10.

22. Rachel Rosen, Kristen Hart, and Samuel Nurko, Does Reflux Monitoring With Multichannel Intraluminal Impedance Change Clinical Decision Making? J Pediatr Gastroenterol Nutr 2011;52(4):404-407.

23. Wenzl TG, Benninga MA, Loots CM, Salvatore $S$, Vandenplas $Y$. Indications, methodology, and interpretation of combined esophageal impedance- $\mathrm{pH}$ monitoring in children: ESPGHAN EURO-PIG standard protocol. J Pediatr Gastroenterol Nutr 2012;55(2):230-4.

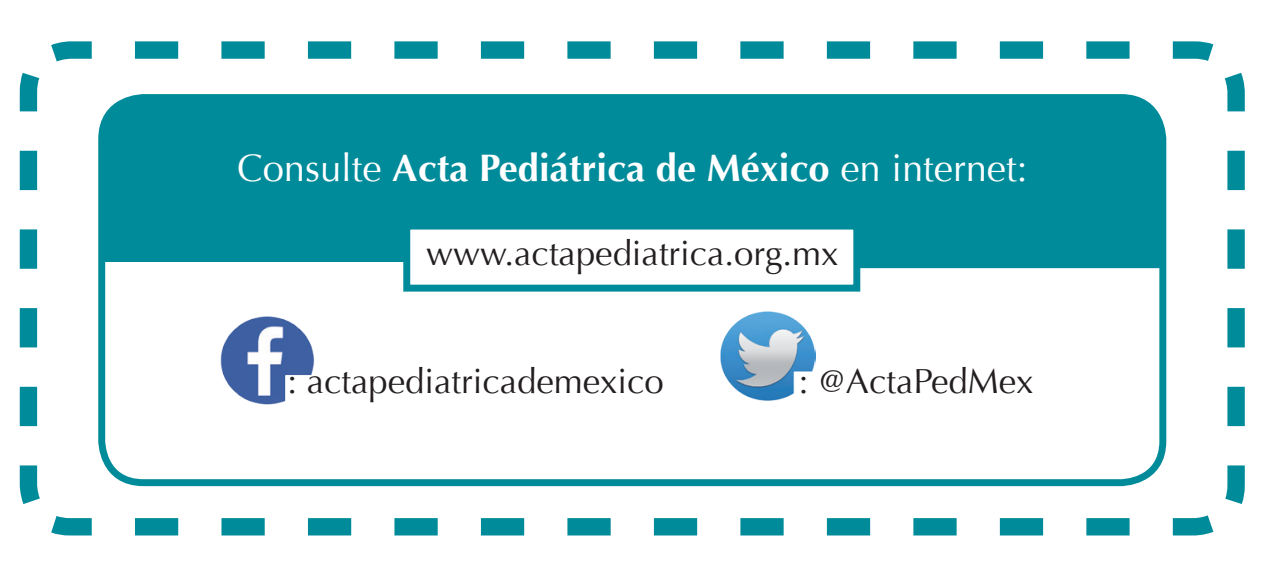

